\title{
A three-pronged approach to tackling warm ischemic injury
}

A team in China has developed a technique to minimize warm ischemic injury (WII), a common occurrence after partial nephrectomy. Their single-surgeon report describes three aspects of the surgery that are needed to reduce WII, which influences the recovery of renal function after treatment.

Changjun Yin's group has focused their efforts on precise segmental renal artery clamping to prevent WII. Rather than conventional main renal arterial clamping, they selectively clamp arteries feeding the tumour. This intuitive approach first requires precise imaging and orientation of the vessels, which they achieve using high-resolution $3 \mathrm{D}$ reconstructive angiography with dual-source CT. Such precise targeting is designed to spare the blood supply to the surrounding tissue and reduce the likelihood of WII.

When accessing the targeted vessels Yin uses a modified retroperitoneal approach, with four laparoscopic ports distributed around the tumour to obtain satisfactory exposure of the infrahilar vessels. The flexibility provided by this approach means that dissection of the artery is possible at the anterior or posterior hilum.

$$
4 \text {... precise [vessel] targeting }
$$

is designed to spare the blood

supply to the surrounding

tissue... 77

Finally, preserving the parenchyma is a priority when excising the tumour. Leaving a 1-2 $\mathrm{mm}$ margin, the surgeon spares any interlobular vessels adhering to the tumour pseudocapsule to maintain blood supply to the healthy tissue.

Retrospective analysis of the 125 patients who underwent unilateral kidney tumour resection by Yin showed that accuracy of orientating the feeding arteries was almost 94\%. Furthermore, a higher number of clamps predicted large tumours with striding locations and endophytic patterns, which might prove useful as a prognostic factor in the future. The average surgery time was only $87 \mathrm{~min}$, and in the 18-month follow-up period, no cases of renal dysfunction, arteriovenous fistula or tumour recurrence were detected, which bodes well for the long-term success of this surgery. Indeed, prospective randomized clinical trial comparing precise segmental with conventional main renal arterial clamping is due to commence later this year. "We hope our work can contribute to the clinical practice for kidney cancer and improve the functional outcomes of patients," Yin concluded.

Mina Razzak

Original article Shao, P. et al. Precise segmental renal artery clamping under the guidance of dual-source computer tomography angiography during laparoscopic partial nephrectomy. Eur. Urol. doi:10.1016/j.eururo.2012.05.056 